\title{
Prediction of Aerodynamic Noise in a Ring Fan Based on Wake Characteristics
}

\author{
Soichi Sasaki ${ }^{1}$, Masaharu Fukuda ${ }^{2}$, Masao Tsujino ${ }^{3}$ and Haruhiro Tsubota ${ }^{3}$ \\ 1. Faculty of Engineering, Nagasaki University, 1-14 Bunkyo-machi, Nagasaki 852-8521, Japan \\ 2. Graduate School of Science and Technology, Nagasaki University, 1-14 Bunkyo-machi, Nagasaki 852-8521, Japan \\ 3. Research Division, Komatsu Ltd., 1200, Manda, Hiratsuka-shi, Kanagawa 254-8567, Japan
}

\begin{abstract}
A ring fan is a propeller fan that applies an axial-flow impeller with a ring-shaped shroud on the blade tip side. In this study, the entire flow field of the ring fan is simulated using a computational fluid dynamics (CFD); the accuracy of the CFD is verified through a comparison with the aerodynamic characteristics of a propeller fan of current model. Moreover, the aerodynamic noise generated by the fan is predicted on the basis of the wake characteristics. The aerodynamic characteristic of the ring fan based on the CFD can represent qualitatively the variation in the measured value. The main flow domain of the ring fan is formed at the tip side of the blade because a blade tip vortex is not formed at that location. Therefore, the relative velocity of the ring fan is increased by the circumferential velocity. The sound pressure levels of the ring fan within a frequency band of less than $200 \mathrm{~Hz}$ are larger than that of the propeller fan. In the analysis of the wake characteristics, it revealed that Karman vortex shedding occurred in the main flow domain in the frequency domain lower than $200 \mathrm{~Hz}$; the aerodynamic noise of the ring fan in the vortex shedding frequency enlarges due to increase in the relative velocity and the velocity fluctuation.
\end{abstract}

Keywords: Fan, Vortex, Aerodynamic Noise, Wake, Internal Flow
CLC number:
Document code: A
Article ID:

\section{Introduction}

In construction machinery, it is necessary to generate a large amount of artificial wind for engine cooling because, unlike in automobiles, cooling fans in the engines cannot expect any help from outside wind [1]. However, increasing the amount of wind in a fan leads to an increase in fan noise. Moreover, since the radiator and engine create resistance, engine cooling fans are not always driven at their highest level of efficiency. Accordingly, air flow at the blade tip side becomes a separated state due to a blade tip vortex. Therefore, in discussions on fan noise in such a case, it is necessary to consider the relationship between a separated flow and aerodynamic noise. In this regard, using a wind tunnel experiment and a numerical simulation, authors previously analyzed the relationship between a separated flow around an airfoil blade and aerodynamic noise [2]. Moreover, author proposed a prediction theory of aerodynamic noise based on the wake characteristics of the blade; and the aerodynamic noise of a forward curved fan has been predicted by implementing this theory [3]. However, to predict the fan noise using this theory, it was necessary to measure the actual flow. On the other hand, it has recently become possible to analyze an entire flow field of a fan using computational fluid dynamics (CFD). Thus, in this study, authors analyze the entire flow field of a propeller fan with a CFD code, and attempt a prediction of the aerodynamic noise generated from the fan on the basis of its wake characteristic. The accuracy

Received: October 2010

Soichi SASAKI: Assistant Professor 
of the aerodynamic characteristics in the simulation is verified through a comparison with the measured values. The influence of a wake on the aerodynamic noise generated from propeller fans is discussed primarily on the basis of the flow field in CFD.

\begin{tabular}{llll}
\hline \multicolumn{2}{c}{ Nomenclature } & $U$ & circumferential velocity $(\mathrm{m} / \mathrm{s})$ \\
$C_{L}$ & lift coefficient & $w$ & relative velocity $(\mathrm{m} / \mathrm{s})$ \\
$D$ & diameter of impeller $(\mathrm{m})$ & $y^{+}$ & normalized distance \\
$D$ hub & diameter of hub $(\mathrm{m})$ & Greek letters & \\
$D^{*}$ & width of wake $(\mathrm{m})$ & $v$ & hub ratio \\
$d$ & scale of vortex $(\mathrm{m})$ & $\varphi$ & flow coefficient \\
$L$ & shaft power $(\mathrm{W})$ & $\psi_{s}$ & static pressure coefficient \\
$L_{p}$ & sound pressure level $(\mathrm{dB})$ & $\rho$ & density of air $\left(\mathrm{kg} / \mathrm{m}^{3}\right)$ \\
$N$ & rotational speed $(\mathrm{r} / \mathrm{min})$ & $\lambda$ & power coefficient \\
$P_{S}$ & static pressure $(\mathrm{Pa})$ & $\eta$ & efficiency \\
$p_{0}$ & base sound pressure $(20 \mu \mathrm{Pa})$ & Subscripts & \\
$Q$ & volume flow rate $\left(\mathrm{m}^{3} / \mathrm{s}\right)$ & velocity fluctuation \\
$S_{t}$ & Strouhal number & & \\
\hline
\end{tabular}

\section{Experimental Setup}

\section{Experimental apparatus}

Figure 1 shows a given impeller of a fan, the main dimensions of which are listed in Table 1. Figures 1 (a) and 1 (b) are the impellers of propeller fan and ring fan, respectively. The sizes of the blades used in the two impellers are the same except for the existence of the shroud assembly in the blade tip side of the ring fan. The overall size of the fan is defined on the basis of the diameter of the impeller. The hub ratio $(v=\mathrm{D}$ hub/D) becomes 0.424 .

A schematic view of the experimental apparatus is shown in Fig. 2. The height and width of the apparatus

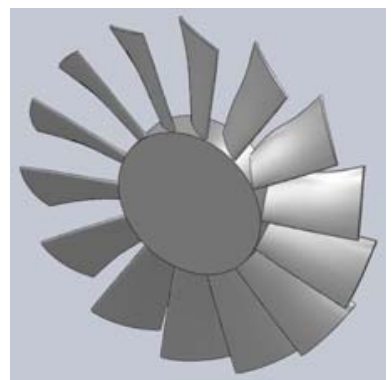

(a) Propeller fan

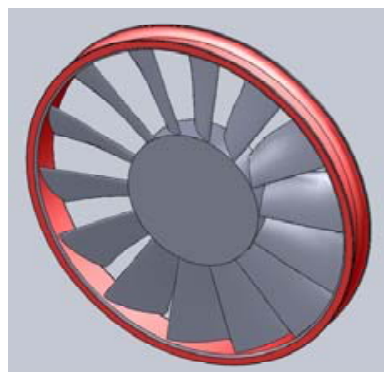

(b) Ring fan
Fig. 1 Test impeller

Table 1 Main dimensions of the impeller

\begin{tabular}{|c|c|c|}
\hline & Propeller Fan & Ring Fan \\
\hline$D(\mathrm{~m})$ & \multicolumn{2}{|c|}{0.613} \\
\hline$D_{\text {hub }}(\mathrm{m})$ & \multicolumn{2}{|c|}{0.260} \\
\hline$v=D_{\text {hub }} / D$ & \multicolumn{2}{|c|}{0.424} \\
\hline Shroud & without shroud & with shroud \\
\hline
\end{tabular}

are $1 \mathrm{~m}$ each, while its total length is about $4 \mathrm{~m}$. The dynamic pressure at a position $600 \mathrm{~mm}$ upstream from the criterion position of the impeller is measured using a pitot tube; the flow rate of the fan is decided by the dynamic pressure. The flow rate is controlled using a
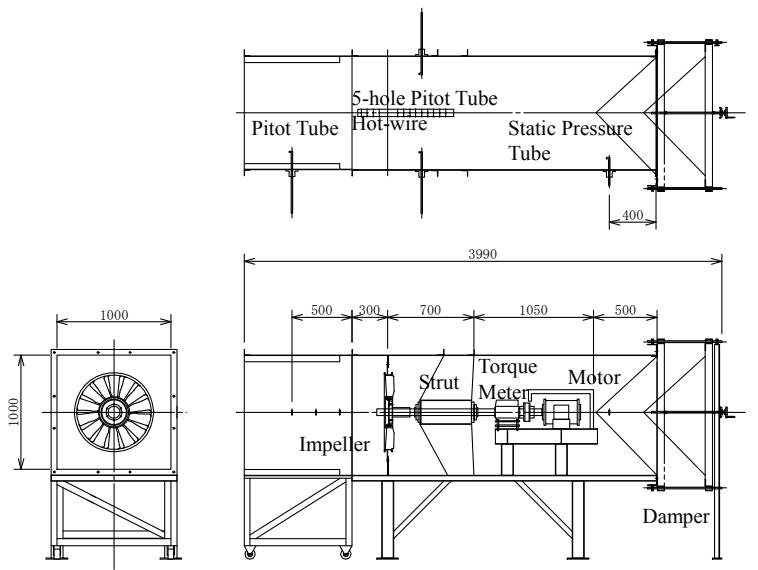

(a) Measurement of aerodynamic characteristics

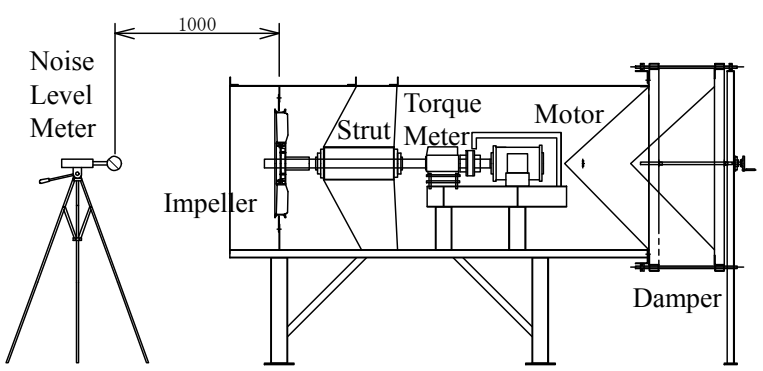

(b) Measurement of fan noise

Fig. 2 Experimental apparatus 
damper provided at the exit of the body. The static pressure of the fan is measured using a static pressure tube provided $400 \mathrm{~mm}$ upstream from the exit of the body. The shaft torque of the motor is measured using a torque meter (Ono-Sokki; SS-500); the efficiency of the fan can be estimated on the basis of the ratio of shaft power to theoretical power. The flow coefficient $\varphi$, static pressure coefficient $\psi_{s}$, power coefficient $\lambda$, and efficiency $\eta$ are summarized in Eq. (1).

$\varphi=4 Q / \pi\left(1-v^{2}\right) D^{2} U$

$\psi_{s}=2 P_{s} / \rho U^{2}$

$\lambda=8 L / \rho \pi\left(1-v^{2}\right) D^{2} U^{3}$

$\eta=\varphi \psi_{t} / \lambda$

Unsteady flow phenomena are measured using a hot-wire anemometer. The insertion hole of the hot-wire probe was set at a position $50 \mathrm{~mm}$ from the rear of the trailing edge of the blade.

Figure 3 shows an overall view of the fan model used for numerical simulation. The CFD code of SCRYU/Tetra, produced by Software Cradle Co., Ltd., was used in the numerical simulation. Mechanisms such as motors and bearings set at the downstream of the fan are omitted in the model. The body and entire length of the apparatus were designed using the same sizes as a real apparatus. The rotational speed in the driving shaft was $1200 \mathrm{rpm}$. The minimum grid width on the blade surface was chosen as $y^{+}=100$ on the basis of a realistic computation time (CPU; Intel Xeon $54102.33 \mathrm{G} \mathrm{Hz}$, Memory; 2GB). About four million grid elements were used to solve the whole flow field in the fan. A total pressure of $0 \mathrm{~Pa}$ was given to the inlet boundary of the model, while a static pressure of $0 \mathrm{~Pa}$ was given to the outlet side.

\section{Fan Noise Prediction Theory}

According to Ref. [3], aerodynamic noise generated from a blade element of an impeller is given as Eq. (2).

$\bar{p}=\frac{\rho w^{3} S_{t} \lambda d \overline{C_{L}}}{4 a_{0} r}$,

where

$\overline{C_{L}}=2 \kappa \pi \frac{\overline{w^{\prime}}}{w}$,

where $\kappa$ is the intermittency factor of the wake vortex shedding, and is given as $\kappa=0.2806 d / D$, theoretically. The sound pressure level of the fan is then expressed as Eq. (3).

$L_{p}=10 \log _{10}\left(\frac{p^{2}}{p_{0}^{2}}\right)$,

where $p_{0}$ is the base sound pressure $(20 \mu \mathrm{Pa})$. According

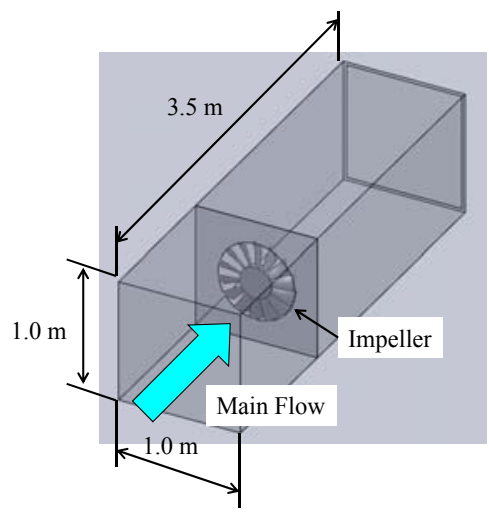

(a) 3D model of the fan

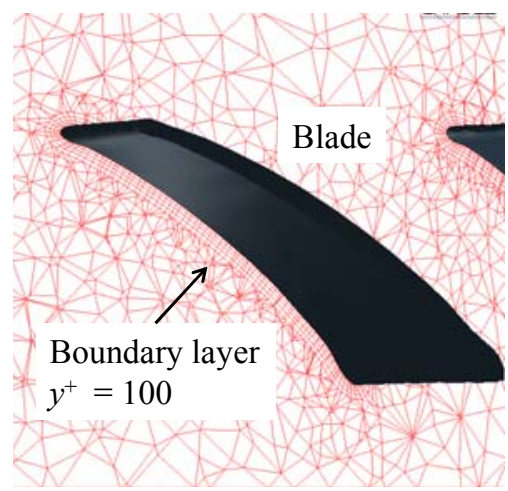

(b) Boundary layer

Fig. 3 CFD model of the propeller fan

to Ref. [2], which details a wind tunnel experiment on the wake characteristics of an airfoil blade, the relationship between the width of wake $D^{*}$ in a separated flow and the scale of vortex $d$ becomes $d=0.8 D^{*}$. From the abovementioned relationship, the sound pressure level generated from the wake vortex can be predicted from the relative velocity $w$, normalized velocity fluctuation $w^{\prime} / w$, and the width of wake $D^{*}$.

\section{Results and Discussion}

Figure 4 shows the aerodynamic characteristics analyzed using the experimental fluid dynamics (EFD) and CFD, where $\circ$ illustrates the characteristics of the propeller fan, and - shows those of the ring fan. The static pressure coefficient of the ring fan in the EFD was higher than in the propeller fan over a wide flow rate domain. Accordingly, the efficiency of the EFD in the vicinity of the maximum efficiency point $(\varphi=0.4)$ of the ring fan becomes about $12 \%$ higher than in the propeller fan. The aerodynamic characteristic of the ring fan based on the CFD can be represented by the variation in the measured value; however, the estimated flow rate of the 


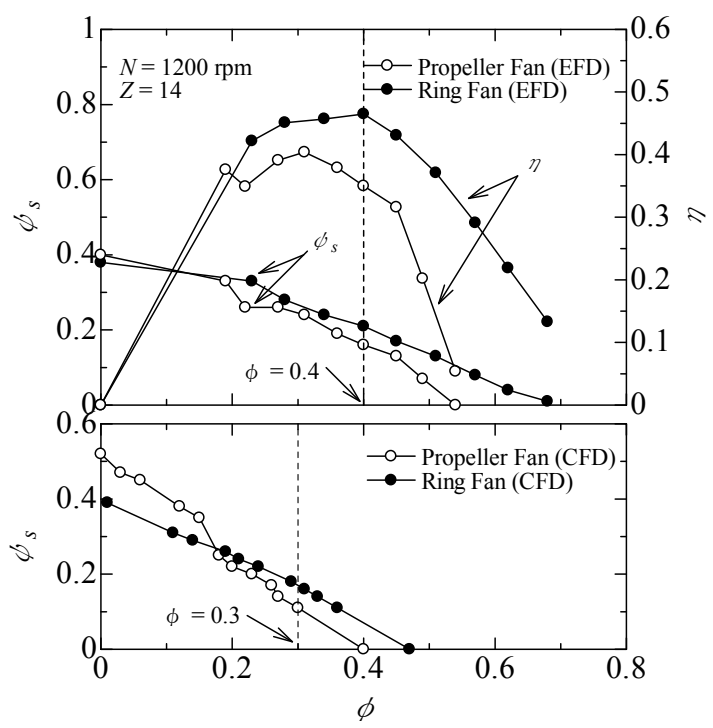

Fig. 4 Aerodynamic characteristics

CFD is about $30 \%$ lower than the EFD. When the ratio of the flow rate in EFD/CFD is taken into consideration, the flow coefficient of the ring fan in the CFD becomes around $\varphi=0.3$.

The flow patterns in the wake are shown in Fig. 5. The vertical plane and horizontal plane are the velocity and pressure distributions, respectively. In the velocity distribution, the main flow domain of the propeller fan, which is a high velocity domain, formed at the $200 \mathrm{~mm}<$ $\mathrm{r}<300 \mathrm{~mm}$ span of the blade. On the other hand, the main flow domain of the ring fan was created at the vicinity of the blade tip side than that of the propeller fan. For the pressure distribution, the pressure of the ring fan in the wake is higher than in the propeller fan. Moreover, it can be observed that the low pressure domain that formed at the blade tip side of the propeller fan did not form at the blade tip side of the ring fan. It is considered that because the leakage flow at the blade tip side is controlled by the shroud assembly of the ring fan, a tip vortex was not formed there.

In Fig. 6, the spectra of the sound pressure level of the two types of fans are compared. The thin line is the spectra of the propeller fan, while the thick line is that of the ring fan. A discrete frequency noise (DFN) was generated at $280 \mathrm{~Hz}$, synchronized with the blade passing frequency. The DFN was generated by the rotation of the moving blade or the interference with the wake of the impeller and motor strut. The sound pressure level of the ring fan is higher than in the propeller fan over a wide frequency domain of less than $200 \mathrm{~Hz}$.

The distributions of relative velocity toward the span direction in the wake are shown in Fig. 7. The

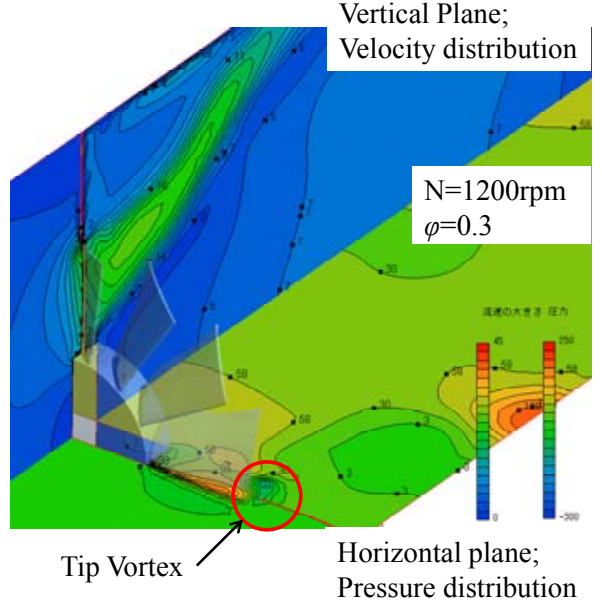

(a) Propeller fan

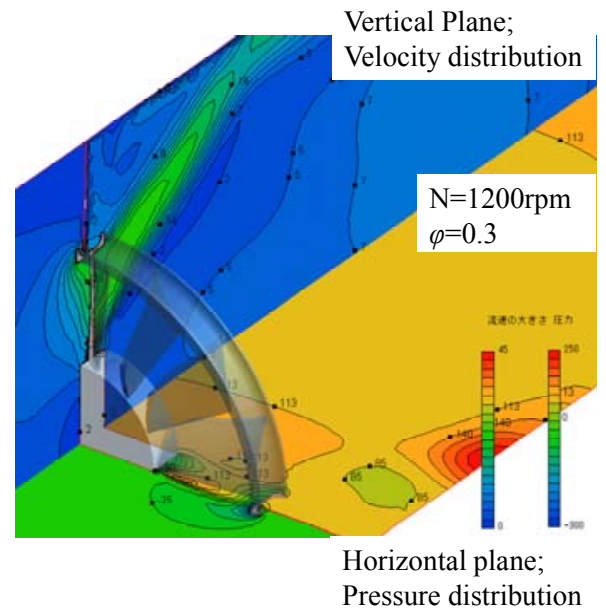

(b) Ring fan

Fig. 5 Flow pattern in the wake of each fan

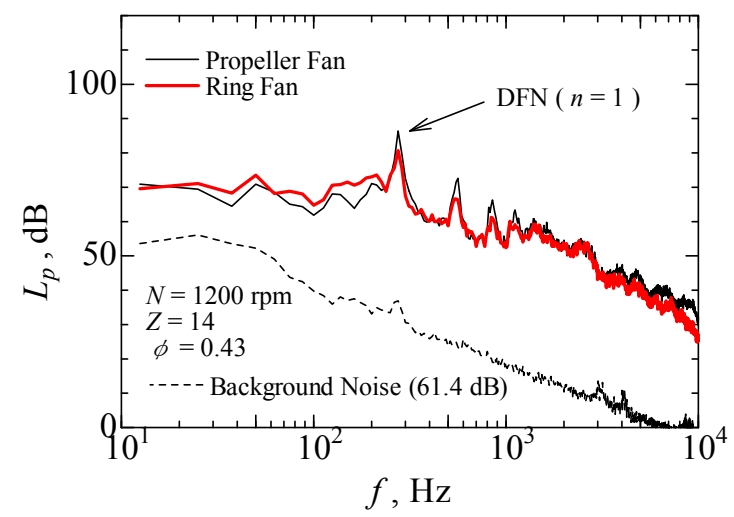

Fig. 6 Spectra of the sound pressure levels 


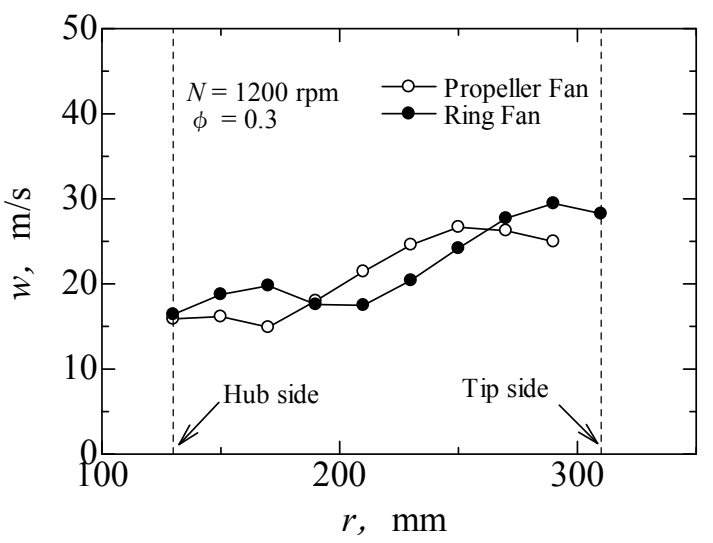

Fig. 7 Distribution of the relative velocities in the span direction

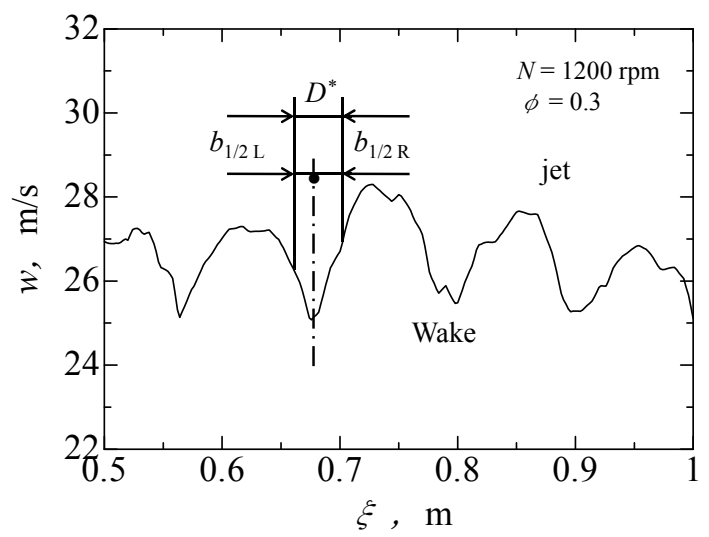

Fig. 8 Distribution of the relative velocities in the circumference direction of the propeller fan

measurement position for the velocity was set at $50 \mathrm{~mm}$ from the rear of the trailing edge of the blade. The relative velocity of the propeller fan reached its maximum level at the vicinity of $r=250 \mathrm{~mm}$; on the other hand, the velocity in the ring fan became its maximum level at the vicinity of the blade tip side. The maximum velocity of the ring fan was higher than that of the propeller fan. Since a tip vortex was not formed at the blade tip side in the ring fan, the main flow domain of the ring fan was located within the vicinity of the blade tip side. Therefore, the relative velocity of the ring fan becomes higher than in the propeller fan because of the influence of high circumferential velocity at the blade tip side.

The distribution of the relative velocity in the direction of the circumference is shown in Fig. 8. This is the distribution at $r=250 \mathrm{~mm}$. The horizontal axis is the distance in the circumference direction of the impeller. In

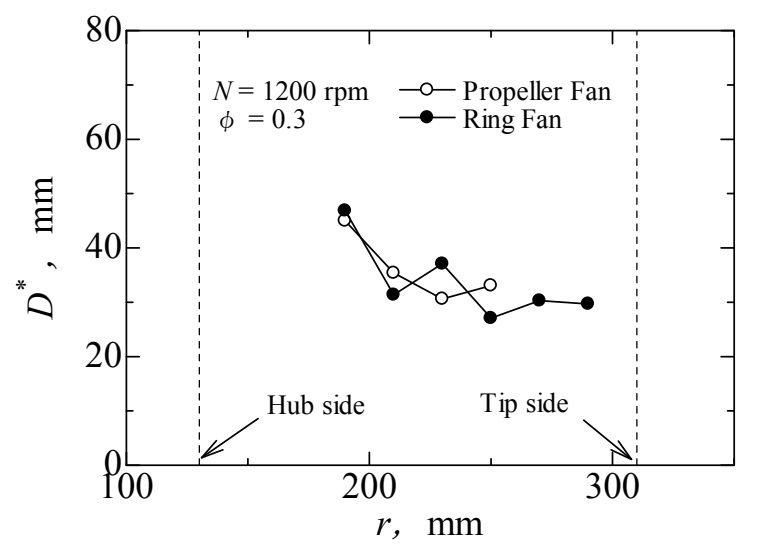

Fig. 9 Distribution of the wake widths in the span direction

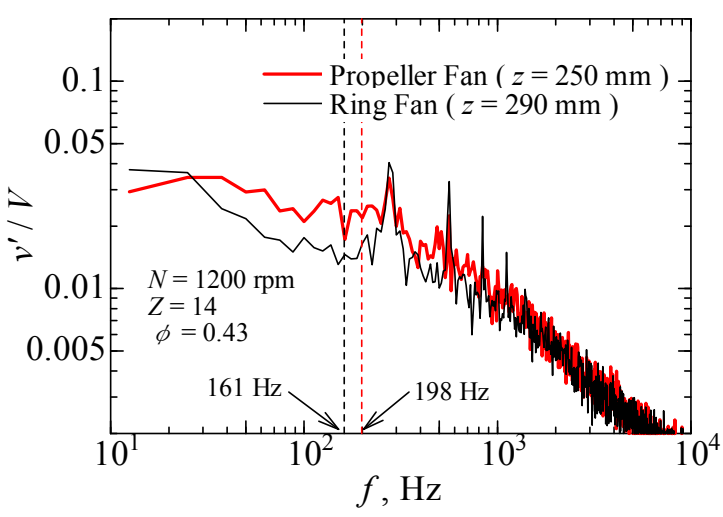

Fig. 10 Spectra of the velocity fluctuation

the distribution of this relative velocity, wake and jet flows were alternately formed. The width of the wake in one pitch is estimated on the scale which is the sum of the half-width at the pressure surface and suction surface sides (see $D^{*}$ in Fig. 8).

In Fig. 9, the wake widths in the direction of the blade span are compared. A large difference did not occur in the distribution of scale in these fans. For each fan, the width on the hub side extended, while the width at the blade tip side narrowed. The frequency of the vortex shedding is estimated on the basis of the wake characteristics using the relationship of Strouhal number $\left(S_{t}=f D^{*} / w \doteqdot 0.202\right)$; the vortex shedding frequency of the ring fan is $198 \mathrm{~Hz}$, while in the propeller fan it was $161 \mathrm{~Hz}$.

The spectra of the velocity fluctuation measured using the hot-wire anemometer is presented in Fig. 10. The velocity fluctuation was measured at the position where 


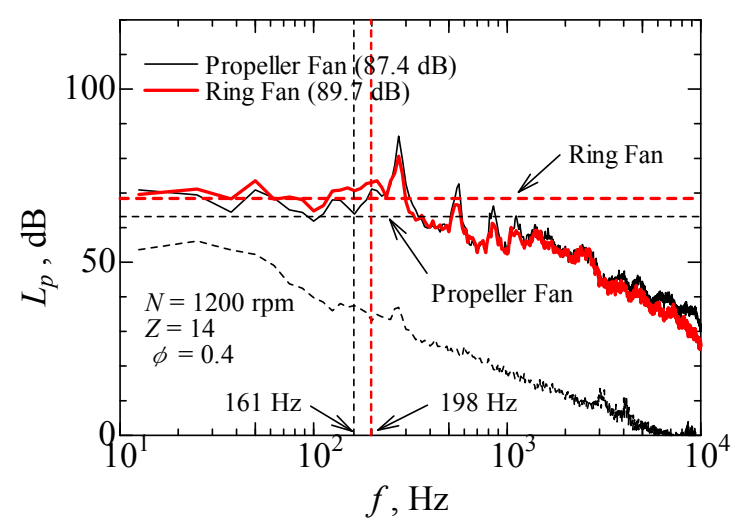

Fig. 11 Comparison of sound pressure levels with their predicted values

the relative velocity reaches its maximum level. Within the vicinity of the vortex shedding frequency, the velocity fluctuation of the ring fan is larger than that of the propeller fan. The predicted fan noise on the basis of the wake characteristics and the measured fan noise are compared in Fig. 11. The intersection of the broken line is predicted values. The measured fan noise of the ring fan within the vicinity of the vortex shedding frequency is higher than in the propeller fan. According to these analyses of the wake characteristics, the sound pressure level is increased by the relative velocity and the velocity fluctuation since the main flow domain of the ring fan formed at the blade tip side.

\section{Conclusions}

Authors analyzed the flow fields in two types of propeller fans on the basis of numerical simulation and measurements. From the results of a discussion on the influence of the wake characteristics on the fan noise, the following conclusions were obtained.
[1] The aerodynamic characteristics of the fans based on CFD can represent qualitatively the variation in the measured value; however, the flow rate of the CFD was about $30 \%$ lower than the measured value.

[2] Since a tip vortex in the ring fan did not form at the blade tip side, the main flow domain of the ring fan was located in the vicinity of the blade tip side. Therefore, the relative velocity of the ring fan becomes higher than that of the propeller fan because of the influence of the high circumferential velocity at the blade tip side.

[3] The sound pressure level of the ring fan is higher than that of the propeller fan over a wide frequency domain of less than $200 \mathrm{~Hz}$. From the results of the analysis on the wake characteristics, it is inferred that wake vortex shedding occurred in these fans within this frequency domain.

[4] According to the analysis on the wake characteristics, the sound pressure level in the ring fan increased due to the relative velocity and velocity fluctuation because the main flow domain formed at the blade tip side.

\section{Acknowledgement}

The authors wish to acknowledge the support of the Harada Memorial Foundation for this study.

\section{References}

[1] Tsubota, H.: Research and Development of Ring Fan, Komatsu Technical Report (in Japanese), vol. 53 , no. 159 , pp. 2-9, (2007).

[2] Sasaki, S. et al.: Influence of Separated Vortex on Aerodynamic Noise of an Airfoil Blade, Journal of Thermal Science, vol. 19, no. 1, pp. 60- 66, (2010).

[3] Sasaki, S. et al.: Application of Wake Characteristics to Prediction of Broadband Noise of a Multiblade Fan, Journal of Fluid Science and Technology, vol. 3, no. 6, pp. 814-825, (2008). 